\title{
Theoretical Overview of Lorentz and CPT Violation
}

\author{
Don Colladay
}

New College of Florida, Sarasota, FL

\begin{abstract}
In this talk, I discuss some recent theoretical progress concerning the Lorentz- and CPTviolating extension of the standard model. The results summarized include the development of an explicit connection between noncommutative field theory and the standard model extension, placement of new bounds in the photon sector, calculation of one-loop renormalization beta functions in QED, and an analysis of field redefinitions.
\end{abstract}

\section{OVERVIEW}

For over ten years now there has been active interest in the possibility that more fundamental theories may induce small violations of Lorentz and CPT invariance into the standard model at levels accessible to high precision experiments [1]. The original motivation for the idea arose from string theory [2] in which higher order field interactions due to the non-local nature of strings may modify the Lorentz properties of the vacuum. The general mechanism developed to model this effect at the level of the standard model is spontaneous symmetry breaking in which tensor fields attain a nonvanishing expectation value in the vacuum at low energies. In fact, the idea of a generic spontaneous symmetry breaking mechanism can be applied to generic fundamental theories that reduce to the standard model at low energies.

A Standard Model Extension (SME) that includes all possible terms arising from a generic spontaneous symmetry breaking mechanism of this type has been constructed [3]. These terms may violate Lorentz and/or CPT invariance. The framework is that of conventional quantum field theory in which standard techniques can be implemented to calculate the effects of Lorentz and CPT violation on physical processes. Sensitive experimental tests of Lorentz and CPT symmetry include accelerator experiments [4, 5], low-energy atomic experiments [6, 7, 8], and astrophysical tests [9, 10].

There is a deep connection between Lorentz invariance and CPT symmetry from the well-known CPT theorem as well as the more recent result by Greenberg that CPT violation in fact requires a violation of Lorentz invariance [11]. In this reference, Greenberg also considers a generic field theory in which one tries to introduce a separate mass for the particle and antiparticle states. He shows that there is necessarily a violation of locality as well as a violation of coordinate Lorentz invariance in such theories. This implies that different observers would not be able to make consistent calculations in such a theory. Therefore, bounds of CPT symmetry can be interpreted as bounds on Lorentz invariance. Note that Lorentz violation does not necessarily imply CPT violation as can be seen from explicit terms of this type in the SME. 
To begin, I will discuss the construction of the SME as well as some motivation for its development. Following this introduction, I will give a summary of four theoretical papers that have significantly developed the framework involved. Other papers in this proceedings include an analysis of a supersymmetric generalization [12] and Lorentz violation induced time variation of physical constants [13].

\section{INTRODUCTION TO LORENTZ AND CPT VIOLATION}

As mentioned in the previous section, there is good theoretical motivation for the possibility that Lorentz and CPT invariance may be an approximation at low-energy scales. In addition to the theoretical motivation, many experiments that involve high precision tests of relativity require a common framework within which to compare bounds on various types of physical measurements. Having an explicit theory in terms of the fundamental fields of the standard model allows different experiments to compare bounds on parameters and get a quantitative handle on effects that they are sensitive to. For example, the photon spectropolarimetry measurements place a bound on Lorentz violation in the photon sector that is sixteen orders of magnitude more stringent than the Gamma ray burst and pulsar data [10]. This will be discussed later in the talk.

The mechanism used to generate the SME is spontaneous symmetry breaking applied to fields with tensor indices. This mechanism is analogous to the Higgs mechanism in which a scalar field gains a vacuum expectation value and generates masses for the standard model particles. In the case of a tensor field $\left(B^{\mu}(x)\right.$ for example) containing Lorentz indices, a nonzero expectation value will select out a specific direction in spacetime breaking Lorentz invariance spontaneously. Potentials for tensor fields are absent in conventional renormalizable field theories but can occur in low-energy field expansions of more fundamental theories such as string field theory [2]. Couplings between these tensor fields and standard model particles (such as $B_{\mu} \bar{\psi} \gamma^{5} \gamma^{\mu} \psi$ ) induce violations of Lorentz invariance in the low-energy effective theory due to the spontaneous symmetry breaking (for example $\left\langle B_{\mu}\right\rangle \neq 0$ ).

The SME [3] consists of all such terms arising from couplings between standard model fields and background tensor fields. In general, there are terms in the SME that are nonrenormalizable and terms that violate gauge symmetries. To simplify, it is useful to restrict this very general theory of Lorentz and CPT violation to satisfy $S U(3) \times S U(2)$ $\times U(1)$ gauge invariance and power counting renormalizability. Restricting further to spacetime independent expectation values generates the minimal SME that is useful for quantifying leading order corrections to experiments.

As an example, the QED sector of the minimal SME is given here. The QED extension is obtained by restricting the minimal SME to the electron and photon sectors. The electron terms are ${ }^{1}$

$$
\mathscr{L}_{e}=\frac{1}{2} \imath \bar{\psi} \Gamma^{v} \stackrel{\leftrightarrow}{D}_{v} \psi-\bar{\psi} M \psi
$$

\footnotetext{
${ }^{1}$ Additional correction terms consistent with $U(1)$ symmetry of the electromagnetic sector, but not with $\mathrm{SU}(2)$ symmetry of the full electroweak sector are often also included. With this relaxed condition, the terms $e^{v}+i f^{v} \gamma^{5}+\frac{1}{2} g^{\lambda \mu v} \sigma_{\lambda \mu}$ may be added into the definition of $\Gamma^{v}$.
} 
where $\Gamma$ and $M$ denote

$$
\begin{gathered}
\Gamma^{v}=\gamma^{v}+c^{\mu v} \gamma_{\mu}+d^{\mu v} \gamma_{5} \gamma_{\mu}, \\
M=m+a_{\mu} \gamma^{\mu}+b_{\mu} \gamma_{5} \gamma^{\mu}+\frac{1}{2} H^{\mu v} \sigma_{\mu v} .
\end{gathered}
$$

The parameters $a, b, c, d$, and $H$ are fixed background expectation values of tensor fields that break conventional particle Lorentz symmetry. The photon terms are

$$
\mathscr{L}_{\gamma}=-\frac{1}{4} F^{\mu v} F_{\mu v}-\frac{1}{4}\left(k_{F}\right)_{\kappa \lambda \mu v} F^{\kappa \lambda} F^{\mu v}+\frac{1}{2}\left(k_{A F}\right)^{\kappa} \varepsilon_{\kappa \lambda \mu v} A^{\lambda} F^{\mu v},
$$

where $k_{F}, k_{A F}$ are the fixed background tensor fields.

Next, I will discuss several new theoretical results that have emerged over the past two years that are associated with the general SME.

\section{CONNECTION TO NONCOMMUTATIVE FIELD THEORY}

There has been much interest recently in the possibility that the coordinates used to parameterize the standard model fields may not commute. Such a situation can arise naturally in the low energy limit of certain string theories [14]. In this case, the nonvanishing commutators can take the special form

$$
\left[x^{\mu}, x^{v}\right]=i \theta^{\mu v}
$$

where the parameters $\theta$ violate Lorentz invariance as they are fixed background parameters. It has been shown [15] that any realistic theory of noncommutative geometry must be physically equivalent to a subset of the SME. The proof relies on the existence of a correspondence between the fields on noncommutative coordinates and conventional fields on commutative coordinates called the Seiberg-Witten map [16]. The result follows by using this map to identify the appropriate Lorentz-violating extension parameters that are present in the resulting theory. More recently, the map has been applied to the entire standard model [17]. The authors find terms that are consistent with a subset of the SME as expected. As an explicit example, a noncommutative version of QED developed in [15] is discussed here.

One way of implementing the noncommutative structure of the underlying coordinates is to promote an established theory to a noncommutative one using the Moyal $\star$ product representation

$$
(f \star g)(x)=\left.\exp \left(\frac{1}{2} i \theta^{\mu v} \partial_{x^{\mu}} \partial_{y^{v}}\right) f(x) g(y)\right|_{x=y} \quad,
$$

for multiplication of the fields. Noncommutative QED can then be constructed using this multiplication as

$$
\mathscr{L}=\frac{i}{2} \hat{\psi} \star \gamma^{\mu} \overleftrightarrow{\hat{D}}_{\mu} \hat{\psi}-m \overline{\hat{\psi}} \star \hat{\psi}-\frac{1}{4 q^{2}} \hat{F}_{\mu v} \star \hat{F}^{\mu v}
$$

These noncommutative fields $(\hat{\psi}, \hat{A})$ satisfy unconventional gauge transformations and do not correspond to the conventional electrons and photons as described in the framework of conventional quantum field theory. Application of the Seiberg-Witten map [16] 
(to lowest order in $\theta$ )

$$
\begin{gathered}
\hat{A}_{\mu}=A_{\mu}-\frac{1}{2} \theta^{\alpha \beta} A_{\alpha}\left(\partial_{\beta} A_{\mu}+F_{\beta \mu}\right), \\
\hat{\psi}=\psi-\frac{1}{2} \theta^{\alpha \beta} A_{\alpha} \partial_{\beta} \psi,
\end{gathered}
$$

must be used to identify the relevant corrections to the standard electrodynamic fields $(\psi, A)$ in a form that can be directly compared to experimental results. The resulting effective QED theory becomes (to first order in $\theta$ )

$$
\begin{aligned}
\mathscr{L}=\quad & \mathscr{L}_{0}-\frac{i}{8} q \theta^{\alpha \beta} F_{\alpha \beta} \bar{\psi} \gamma^{\mu} \stackrel{\leftrightarrow}{D_{\mu}} \psi+\frac{1}{4} i q \theta^{\alpha \beta} F_{\alpha \mu} \bar{\psi} \gamma^{\mu} \stackrel{\leftrightarrow}{D_{\beta}} \psi \\
& +\frac{1}{4} m q \theta^{\alpha \beta} F_{\alpha \beta} \bar{\psi} \psi+\left(F^{3} \ldots\right) .
\end{aligned}
$$

The correction terms correspond to nonrenormalizable terms in the $\mathrm{SME}^{2}$. It is possible to examine experiments that occur in constant background electromagnetic fields using $F^{\mu v} \rightarrow f^{\mu v}+F^{\mu v}$ where $f^{\mu v}$ is constant. With this substitution, a specific subset of the terms in the minimal SME are recovered. These are

$$
\mathscr{L}=\mathscr{L}_{0}+\frac{i}{2} c_{\mu \nu} \bar{\psi} \gamma^{\mu} \stackrel{\leftrightarrow}{D^{v}} \psi-\frac{1}{4}\left(k_{F}\right)_{\alpha \beta \gamma \delta} F^{\alpha \beta} F^{\gamma \delta}
$$

with

$$
c_{\mu v}=-\frac{1}{2} q f_{\mu}^{\lambda} \theta_{\lambda v} ; \quad\left(k_{F}\right)_{\alpha \beta \gamma \delta}=-q f_{\alpha}^{\lambda} \theta_{\lambda \gamma} \eta_{\beta \delta}+\cdots \quad .
$$

Atomic experiments in constant $B$ fields can then be used to bound the noncommutative parameters at the level

$$
\left|\theta^{i j}\right|<(10 \mathrm{TeV})^{-2}
$$

Effects of noncommutative geometry on photon propagation in constant background fields have also been considered [19].

\section{ONE LOOP RENORMALIZABILITY OF QED SECTOR}

The next result concerns the explicit analysis of the one-loop renormalizability of the QED sector of the minimal SME [20]. Results included in this reference include:

- Generalized Furry theorem is established showing that the three and four point photon vertices generate a finite contribution to one-loop Green's functions.

- Multiplicative renormalization holds at one loop provided the Lorentz-violating constants are properly renormalized.

- The beta functions have been calculated and the renormalization group was used to examine the running of the violation parameters.

\footnotetext{
2 The possibility of renormalizable terms emerging from loop corrections has been explored [18].
} 
The modified vertices and propagators can be extracted from the lagrangian for extended QED given in equations (1) and (4). For example, the electron-photon vertex will contribute a factor of $-i q \Gamma^{\mu}=-i q\left(\gamma^{\mu}+\varepsilon^{\mu}\right)$ where $\varepsilon^{\mu}$ is a small perturbative correction that depends on the Lorentz and CPT violating terms.

The running of the coupling constants were calculated using renormalization group techniques. They are found to depend on various anomalous powers of the parameter

$$
Q(\mu)=1-\frac{q_{0}^{2}}{6 \pi^{2}} \ln \frac{\mu}{\mu_{0}},
$$

that controls the usual running of the QED charge according to $q=Q^{-1} q_{0}$. In the above expressions, $\mu$ is the renormalized mass scale while $\mu_{0}$ is a reference scale at which the boundary conditions on the parameters are applied. As an example, the $a$ parameters run according to

$$
a^{\mu}=a_{0}^{\mu}-m_{0}\left(1-Q^{9 / 4}\right) e_{0}^{\mu}
$$

while the $c$ parameters run as

$$
c^{\mu v}=c_{0}^{\mu v}-\frac{1}{3}\left(1-Q^{-3}\right)\left(c_{0}^{\mu v}+c_{0}^{v \mu}-\left(k_{F}\right)_{0}^{\mu v \alpha}\right) .
$$

If the parameters are assumed to be unified at the Planck scale, a naive running to low energies indicates that the parameters can differ by 2 to 3 orders of magnitude at the low-energy scale. This result emphasizes the necessity of independently measuring all of the parameters that control Lorentz violation as they may be very different in size.

\section{NEW BOUNDS ON PARAMETERS IN THE PHOTON SECTOR}

Various cosmological experiments have already placed stringent bounds on the CPT violating photon terms [9]. In addition, there are theoretical reasons to suggest that these terms are exactly zero [3, 21]. However, there is no such theoretical bias concerning the CPT even photon terms and they have recently been analyzed in [10]. In this reference, an explicit analogy is constructed between photon propagation in a classical anisotropic medium and photon propagation in a Lorentz-violating background field. The formalism provides a clean way of extracting bounds on all of the CPT-even parameters using both astrophysical and lab based photon propagation experiments.

The relevant term $k_{F}$ modifies the Maxwell equations according to

$$
\partial_{\alpha} F_{\mu}^{\alpha}=-\left(k_{F}\right)_{\mu \alpha \beta \gamma} \partial^{\alpha} F^{\beta \gamma} \quad, \quad \partial_{\mu} \tilde{F}^{\mu v}=0
$$

Note that the homogeneous equations are unmodified as these only depend on the definition of $F^{\mu v}=\partial^{\mu} A^{v}-\partial^{v} A^{\mu}$. To construct the analogy with anisotropic media, fields $\vec{D}$ and $\vec{H}$ are defined according to

$$
\left(\begin{array}{c}
\vec{D} \\
\vec{H}
\end{array}\right)=\left(\begin{array}{cc}
1+\kappa_{D E} & \kappa_{D B} \\
\kappa_{H E} & 1+\kappa_{H B}
\end{array}\right)\left(\begin{array}{c}
\vec{E} \\
\vec{B}
\end{array}\right)
$$


where the various $\kappa$ quantities are $2 \times 2$ constant matrices depending on the $k_{F}$ parameters. Using this definition, the modified Maxwell equations take the conventional form of

$$
\begin{array}{lll}
\vec{\nabla} \times \vec{H}-\partial_{0} \vec{D}=0 & , \quad \vec{\nabla} \cdot \vec{D}=0 \\
\vec{\nabla} \times \vec{E}+\partial_{0} \vec{B}=0 & , \quad \vec{\nabla} \cdot \vec{B}=0
\end{array}
$$

The form of these equations implies that standard techniques can be used to solve the equations of motion.

The violation terms can be divided into ones that cause birefringence and ones that do not. Birefringence measurements can be performed with high sensitivity using astrophysical measurements, while the other terms can be bounded using various resonant cavity experiments. I will focus here on two types of astrophysical tests analyzed in [10]. The first involves gamma ray bursts and pulsars while the second involves spectropolarimetry measurements. Refer to [10] for details concerning the cavity experiments.

Gamma ray bursts and pulsars produce narrow pulses of radiation that propagate large distances. Birefringence implies a velocity difference between the eigenmodes of propagation yielding a spreading of the pulse width in time of $\Delta t \approx \Delta v L$, where $L$ is the distance to the source. Using fifteen different sources, a conservative bound of $\left|k_{F}\right|<3 \times 10^{-16}$ has been placed on CPT-even parameters that cause birefringence.

Much more accurate bounds have been placed on the same parameters using spectropolarimetry data. It is difficult to determine the polarization of most astrophysical sources accurately, so a technique of searching for a specific wavelength dependence in the polarization rotation was implemented. A detailed analysis of the modified Maxwell equations shows that the polarization shift due to birefringence is proportional to the inverse of the wavelength. Combining this fact with the extremely precise time resolution of phase shift time scales yields a bound of $\left|k_{F}\right|<2 \times 10^{-32}$ on the same parameters that are bounded using pulse broadening analysis. This points out the necessity of having a specific theory to calculate explicit bounds on Lorentz symmetry. Spectropolarimetry bounds are far more stringent and they require a detailed knowledge of the form of the modified Maxwell equations as is given in the SME. A simple phenomenological correction to the dispersion relation is not sufficient for a comparable analysis.

\section{FIELD REDEFINITIONS AND LORENTZ VIOLATION}

As the final development discussed in this talk, I will present work done regarding the physical nature of various terms present in the SME [22]. Some terms that are included in the lagrangian (11) can be eliminated using suitable redefinitions of the spinor components. Other terms can be moved to different sectors of the theory. In general, one can define a set of equivalence classes for lagrangians in the SME by relating elements that are connected by redefinitions of the field components. It is not necessary for the redefinitions to be covariant, so the equivalence class of Lagrangians associated to the standard one contains many terms that apparently violate Lorentz or CPT invariance. 
To illustrate the general procedure, we start with the conventional lagrangian for QED

$$
\mathscr{L}[\psi]=\frac{i}{2} \bar{\psi} \gamma^{\mu} \stackrel{\leftrightarrow}{D_{\mu}} \psi-m \bar{\psi} \psi
$$

and apply a redefinition of the spinor field of the form

$$
\psi(x)=[1+f(x, \partial)] \chi(x),
$$

generating a new lagrangian $\mathscr{L}[\chi]$ that may apparently violate Lorentz invariance. As an example, let $f=\frac{1}{2} v_{\mu} \gamma^{\mu}$ where $v_{\mu}$ are real constants. To lowest order in $v$, the lagrangian expressed in terms of $\chi$ is

$$
\mathscr{L}[\chi]=\mathscr{L}_{0}+\frac{i}{2} v_{\mu} \bar{\chi} \stackrel{\leftrightarrow}{D^{\mu}} \chi+m v_{\mu} \bar{\chi} \gamma^{\mu} \chi
$$

If one naively assumes the standard action of $\operatorname{SL}(2, \mathrm{C})$ on the spinors of

$$
\chi^{\prime}\left(x^{\prime}\right)=S(\Lambda) \chi(x)=e^{\frac{i}{4} \omega_{\mu v} \sigma^{\mu v}} \chi(x)
$$

then $\mathscr{L}$ is not covariant. However, it is in fact covariant under the modified action of

$$
\tilde{S}(\Lambda)=e^{-\frac{1}{2} v_{\mu} \gamma^{\mu}} S(\Lambda) e^{\frac{1}{2} v_{\mu} \gamma^{\mu}}
$$

which is related to the standard action by a similarity transformation. This logic can be applied in reverse to conclude that any lagrangian of the form (21) does not in fact violate Lorentz invariance because the fields can be appropriately redefined ${ }^{3}$.

Other redefinitions can involve derivatives and are more complicated. For example, letting $f=C_{\mu v} x^{\mu} \partial^{v}$ yields a transformed lagrangian of (lowest order in $C$ )

$$
\mathscr{L}[\chi]=\mathscr{L}_{0}+C_{\mu v} x^{\mu} \partial^{v} \mathscr{L}_{0}+\frac{i}{2} C_{\mu v} \bar{\chi} \gamma^{\mu} \stackrel{\leftrightarrow}{\partial^{v}} \chi
$$

The second term in this expression is a total derivative up to the term $C_{\mu}^{\mu} \mathscr{L}_{0}$, a term that simply scales the lagrangian. The third term in the expression is the form of the $c$ corrections to $\Gamma$ in equation (11). This transformation is equivalent to a change of coordinates according to

$$
\psi(x)=\left(1+C_{\mu v} x^{\mu} \partial^{v}\right) \chi(x) \approx \chi(x+C \cdot x)=\chi\left(x^{\prime}\right)
$$

where the new coordinates have a metric of $g^{\mu \nu}=\eta^{\mu \nu}+C^{\mu \nu}+C^{v \mu}$. The antisymmetric piece does not alter the metric and corresponds to a conventional Lorentz transformation. The alteration in the form of the lagrangian in this case is compensated by the appropriate element of SL $(2, C)$ for the transformation. The symmetric piece is more interesting

\footnotetext{
${ }^{3}$ Note that an interaction term between a fermion with a free lagrangian of this form and another particle with conventional transformation properties may not be invariant under the redefinition in which case the parameter $v_{\mu}$ would be physical.
} 
as it skews the coordinate system. This can be compensated for using the vierbein formalism of general relativity, but a redefinition of the metric in the photon sector will also be required. Therefore these terms may be eliminated from the electron sector, but they will reappear as corrections in the photon sector. One can understand this result physically as the necessity of using the propagation properties of some particular field to define the coordinate system basis. Once this system is chosen, it is then necessary to measure the propagation properties of other particles with respect to it. Any incompatibility in the interactions will lead to a potentially observable violation of Lorentz invariance in the overall theory.

Terms that can be altered by redefinitions can be reexpressed as appropriate linear combinations such that the terms that are invariant under the field redefintions will correspond to the physically observable parameters. This can be used to significantly simplify models containing Lorentz and CPT violation by reducing the number of parameters that must be included in the calculations. A more complete analysis is performed in [22].

\section{SUMMARY}

In this talk, an overview of recent theoretical progress pertaining to the theory of Lorentz and CPT violation has been presented.

An explicit connection has been made between a subset of the SME and physically realistic theories involving noncommutative field theory. In fact, any theory that violates Lorentz or CPT invariance must reduce to a subset of the general SME provided that corrections to conventional standard model fields are considered and different observers can make consistent calculations regarding physical processes. The extension therefore provides a very robust framework within which violations of Lorentz and CPT symmetry can be analyzed.

One loop renormalizability in the minimal QED extension has been explicitly established. The beta functions indicate a variety of runnings for the various Lorentz- and CPT-violating coupling constants. As a result of the renormalization group analysis, it is possible that parameters that are unified at the Planck scale can differ by a few orders of magnitude at the low-energy scale.

Some apparent violations in the SME can be removed by appropriate field redefinitions. In addition, some parameters can be moved to different sectors using other types of redefinitions. The terms that cannot be altered by redefinitions therefore provide the physically measurable quantities, in accordance with the explicit calculations performed for experimental observables.

\section{REFERENCES}

1. For a summary of recent theoretical work and experimental tests see, for example, CPT and Lorentz Symmetry, V.A. Kostelecký, ed., World Scientific, Singapore, 1999; CPT and Lorentz Symmetry II, V.A. Kostelecký, ed., World Scientific, Singapore, 2002. 
2. V.A. Kostelecký and S. Samuel, Phys. Rev. D 39, 683 (1989); ibid. 40, 1886 (1989); Phys. Rev. Lett. 63, 224 (1989); ibid. 66, 1811 (1991); V.A. Kostelecký and R. Potting, Nucl. Phys. B 359, 545 (1991); Phys. Lett. B 381, 89 (1996); Phys. Rev. D 63, 046007 (2001); V.A. Kostelecký, M. Perry, and R. Potting, Phys. Rev. Lett. 84, 4541 (2000).

3. D. Colladay and V.A. Kostelecký, Phys. Rev. D 55, 6760 (1997); Phys. Rev. D 58, 116002 (1998); V.A. Kostelecký and R. Lehnert, Phys. Rev. D 63, 065008 (2001).

4. KTeV Collaboration, H. Nguyen, in Ref. [1]; OPAL Collaboration, R. Ackerstaff et al., Z. Phys. C 76, 401 (1997); DELPHI Collaboration, M. Feindt et al., preprint DELPHI 97-98 CONF 80 (1997); BELLE Collaboration, K. Abe et al., Phys. Rev. Lett. 86, 3228 (2001); FOCUS Collaboration, J.M. Link et al., hep-ex/0208034

5. D. Colladay and V.A. Kostelecký, Phys. Lett. B 344, 259 (1995); Phys. Rev. D 52, 6224 (1995); Phys. Lett. B 511, 209 (2001); V.A. Kostelecký and R. Van Kooten, Phys. Rev. D 54, 5585 (1996); V.A. Kostelecký, Phys. Rev. Lett. 80, 1818 (1998); Phys. Rev. D 61, 016002 (2000); 64, 076001 (2001); N. Isgur et al. Phys. Lett. B 515, 333 (2001).

6. L.R. Hunter et al., in V.A. Kostelecký, ed., CPT and Lorentz Symmetry, World Scientific, Singapore, 1999; D. Bear et al., Phys. Rev. Lett. 85, 5038 (2000); D.F. Phillips et al., Phys. Rev. D 63, 111101 (2001); M.A. Humphrey et al., Phys. Rev. A 62, 063405 (2000); V.A. Kostelecký and C.D. Lane, Phys. Rev. D 60, 116010 (1999); J. Math. Phys. 40, 6245 (1999); R. Bluhm et al., Phys. Rev. Lett. 88, 090801 (2002).

7. H. Dehmelt et al., Phys. Rev. Lett. 83, 4694 (1999); R. Mittleman et al., Phys. Rev. Lett. 83, 2116 (1999); G. Gabrielse et al., Phys. Rev. Lett. 82, 3198 (1999); R. Bluhm et al., Phys. Rev. Lett. 82, 2254 (1999); Phys. Rev. Lett. 79, 1432 (1997); Phys. Rev. D 57, 3932 (1998).

8. B. Heckel in Ref. [1]; R. Bluhm and V.A. Kostelecký, Phys. Rev. Lett. 84, 1381 (2000).

9. S.M. Carroll, G.B. Field, and R. Jackiw, Phys. Rev. D 41, 1231 (1990).

10. V.A. Kostelecký and M. Mewes, Phys. Rev. Lett. 87 251304(2001); Phys. Rev. D 66056005 (2002).

11. O.W. Greenberg, Phys. Rev. Lett. 89, 2316021 (2002).

12. M. Berger, this proceedings (hep-ph/0212353).

13. R. Lehnert, this proceedings; V. A. Kostelecký, R. Lehnert, M. Perry, (astro-ph/0212003).

14. A. Connes, M. Douglas, and A. Schwartz, JHEP 02003 (1998); For recent reviews see N.A. Nekrasov, hep-th/0011095, A. Konechny and A. Schwartz, hep-th/0012145, J. A. Harvey, hep-th/0102076

15. S. Carroll et al., Phys. Rev. Lett. 87, 141601 (2001).

16. N. Seiberg and E. Witten, JHEP 09032 (1999); A.A. Bichl et al., hep-th/0102103

17. X. Calmet et al., Eur. Phys. J. C23 363 (2002).

18. A. Anisimov et al., hep-ph/0106356

19. Z. Guralnik et al., Phys. Lett. B 517, 450 (2001);

20. V.A. Kostelecký, C. Lane, and A. Pickering, Phys. Rev. D 65056006 (2002).

21. V.A. Kostelecký and R. Lehnert, Phys. Rev. D 63, 065008 (2001).

22. D. Colladay and P. McDonald, J. Math. Phys. 433554 (2002). 